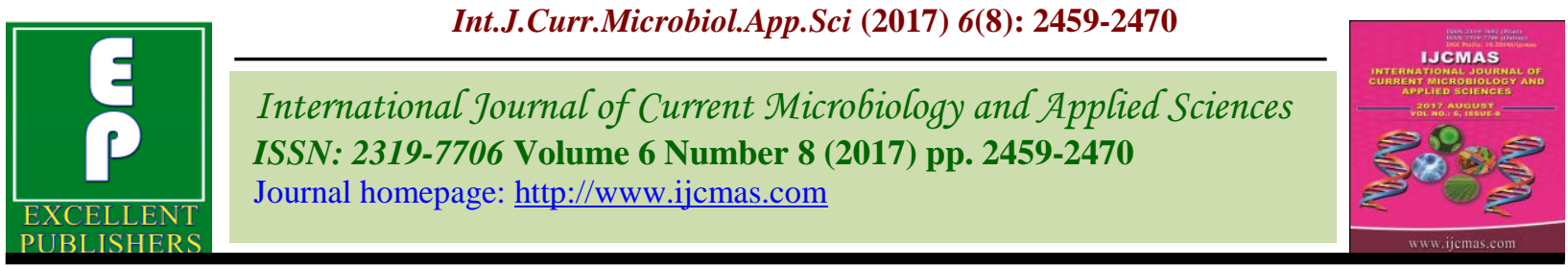

Original Research Article

https://doi.org/10.20546/ijcmas.2017.608.291

\title{
Genetic Studies in Amaranthus Germplasm
}

\author{
Ishwar Singh Diwan*, Neeraj Shukla and Vivek Kurrey \\ Department of Horticulture, Indira Gandhi Krishi Vishway Vidyalaya, \\ Krishak Nagar, Raipur (C.G.), India \\ *Corresponding author
}

\section{A B S T R A C T}

An investigation was carried out to study genetic studies in amaranthus germplasm of 10 germplasm of amaranthus at Horticulture Instructional cum Research Farm of Department of Horticulture, Indira Gandhi Krishi Vishwavidyalaya, Raipur (CG) during the period from Octuber, 2014 to February, 2015. The experiment was laid out in the Randomized Complete Block Design with four replications. The data were analyzed to work out the variability, correlation coefficient and path analysis for character viz., number of leaves per plant, leaf length $(\mathrm{cm})$, leaf width $(\mathrm{cm})$, plant height $(\mathrm{cm})$, stem girth $(\mathrm{cm})$, stem weight

Keywords

Genetic, Amaranthus germplasm, Raipur.

Article Info

Accepted:

21 June 2017

Available Online:

10 August 2017 $(\mathrm{gm})$, leaf weight $(\mathrm{gm})$, stem weight $(\mathrm{gm})$, petiole length $(\mathrm{cm})$, panicle length $(\mathrm{cm})$, plant weight $(\mathrm{gm})$, number of cutting, leaf yield (kg/plot), seed yield (gm/plant), 1000 seed weight $(\mathrm{gm})$ and crop duration (sowing to last harvest). The analysis of variance indicated that the mean sum of square due to genotypes were highly significant for all the sixteen characters. Significant mean sum of squares due to leaf yield and attributing characters revealed existence of considerable variability in material studied for improvement of various traits. The highest leaf yield $\mathrm{kg}$ per plot was recorded in genotype 2012/AMVAR4 followed by 2012/AMVAR-7 (17.41 kg/plot), CG Amaranthus-1 (17.36 kg/plot). Moderate estimates of phenotypic and genotypic coefficient of variation for almost all traits except leaf weight show the high genotypic and phenotypic variation indicated that there was high variability offering ample scope for selection of desired variability. Heritability along with genetic advance as percent of mean for all the tested characters indicated that these characters were under additive gene action and there were excellent chances of effective selection for improvement of these traits. Highly significant and positive correlation with leaf yield was observed with plant height, plant weight, stem girth and seed yield, whereas leaf length and petiole length showed negative association with green yield. Path coefficient analysis revealed that stem weight, 1000 seed weight, number of leaves, intermodal length and panicle length were the most important yield components. Selection programme based on these characters is suggested for further improvement.

\section{Introduction}

Amaranthus or chaulai (Amaranthus sp.) is a very popular leafy vegetable in India as well tropical and sub-tropical areas of globe and it can be grown throughout the year because it has very fast growth habit in short duration and large produce of edible matter per unit area, it fits well in a crop rotation hence amaranthus is the largest produced as leafy vegetable in the world. The word "Amaranth" is basically derived from Greek word "Anthos" which means "everlasting" according to Sankaran (1943). Amaranthus tricolor is an important vegetable amaranthus cultivated in India and numbers of domesticated forms are available in India especially in Tamil Nadu, Andhra Pradesh, 
Karnataka and Kerala states. Amranthus preferred for both urban and rural population and it's also considered to be the cheapest leafy vegetable in the market and it could be rightly described as a "poor man's vegetable" (Varalakshmi, 2004). The amaranth can grow under varied soil and agro climatic conditions (Katiyar et al., 2000; Shukla and Singh, 2000) are resistant from heat and drought with no major disease problems. Beside its adaptable nature in various climatic conditions, the amaranth plant also has important nutritional and medicinal properties (Lakshmi and Vimala, 2000). Due to use of amaranth as vegetable and grain crop, it can be a cheap alternative rich source of protein and nutrient for poor people in developing countries (Prakash and Pal 1991; Prakash et al., 1995; Shukla et al., 2006). The protein component of amaranth is quite close to the level recommended by the $\mathrm{FAO} / \mathrm{WHO}$ for balance diet in human. The protein in leaf is up to $14.3 \mathrm{~g} / \mathrm{kg}$ with an average of $12.4 \mathrm{~g} / \mathrm{kg}$ (Shukla et al., 2003, 2006; Prakash and Pal 1991). A large number of necessary minerals have also been reported in amaranthus. The genetic improvement of any crop depends upon the available genetic variability for quantitative traits and its judicious exploitation through efficient breeding methods. India is the center of origin of Amaranthus sp., hence wide genetic variability is available for utilization to developed high yielding varieties. The available variability in trait of amranthus population can be partitioned into heritable and non-heritable parts with the aid of genetic parameters such as GCV, PCV, heritability and genetic advance. Correlation coefficient helps to identify the relative contribution of component characters towards yield. The correlation between yield and a component character may sometimes be misleading. Thus splitting of total correlation into direct and indirect effects would provide a more meaningful interpretation of such association. Path coefficient, which is a standard partial regression coefficient, specifies the cause and effect relationship and measures the relative importance of each variable. Therefore, correlation in combination with path coefficient analysis will be an important tool to find out the association and quantify the direct and indirect influence of one character upon another and in Chhattisgarh state available wide genetic variability of amaranths in the local land races there is urgent need to develop or identify with categorized high yielding varieties. Therefore present investigation under taken to find out the Genetic Studies in Amaranthus Germplasm between various component characters and their showing the possibilities of further genetic improvement in amaranthus.

\section{Materials and Methods}

The experimental material consisted of 10 genotypes of Amaranthus sp. selected from a germplasm collection maintained under AICRP on vegetable crops at the Department of Horticulture, IGKV, Raipur (C.G.). The material was grown in a randomized block design with four replications in winter (October, 2014). The genotypes were raised in flat beds of size $3 \times 2 \mathrm{~m}^{2}$ and evaluated under irrigated condition. Observations were recorded on 10 plants in each replication for the 16 characters viz., plant height, plant weight, number of leaves, leaf length, leaf width, leaf weight, stem weight, stem girth, internodal length, petiole length, panicle length, number of cutting, 1000 seed weight, seed yield, crop duration and leaf yield. The analysis of variance was carried out for each character separately as per method of Panse and Sukhatme (1967). The coefficient of variation for different characters was estimated by formula as suggested by Burton (1952). The heritability was estimated as per the formula suggested by Burton and De Van (1953) and the expected advance was 
calculated by the formula given by Johnson et al., (1955). The Genotypic and phenotypic correlation coefficients were worked out using the formulae suggested by Miller et al., (1958) and path analysis was carried out as suggested by Dewey and Lu (1959) so as to study the direct and indirect contribution of component characters on green yield of amaranthus species.

\section{Results and Discussion}

The analysis of variance for all the characters under study is presented in table 1. These analyses of variance revealed that mean sum of squares due to genotypes were highly significant for all characters. This is an indication of existence of sufficient variability among all the genotypes for leaf yield and its components traits. Similar result reported by Raut et al., (2014).

The observation on ten plants from each genotype of all four replications for leaf yield and its components characters were used for calculating the mean performance. The observations were first averaged for ten plant taken randomly for each genotype in each replication and were later averaged over all the four replications. The mean performance of different genotype are presented character wise in table 2 .

The plant height ranged from $8.39 \mathrm{~cm}$ (2012/AMVAR-2) to $12.15 \mathrm{~cm}$ (CG Amaranthus-1) with an overall mean of 10.10 $\mathrm{cm}$. higher plant height was recorded in the genotype CG Amaranthus-1 $(12.15 \mathrm{~cm})$. The Plant weight ranged from $52.50 \mathrm{gm}$ (2012/AMVAR-1) to 70.50 gm (2012/AMVAR-7) with an overall mean of $62.20 \mathrm{gm}$. Higher plant weight was recorded in 2012/AMVAR-7 (70.50 gm). The number of leaves ranged from 18.56 (2012/AMVAR6) to 26.36 (CG Amaranthus-1) with an overall mean of 20.88. Higher number of leaves was recorded in CG Amaranthus-1 (26.36). The leaf length ranged from $4.83 \mathrm{~cm}$ (2012/AMVAR-1) to $8.98 \mathrm{~cm}$ (Arun) with an overall mean of $6.14 \mathrm{~cm}$. higher leaf length was recorded in Arun $(8.98 \mathrm{~cm})$. The leaf width ranged from $3.45 \mathrm{~cm}$ (2012/AMVAR1) to $5.06 \mathrm{~cm}$ (CG Amaranthus-1) with an overall mean of $4.35 \mathrm{~cm}$. higher leaf width was recorded in CG Amaranthus-1 $(5.06 \mathrm{~cm})$. The leaf weight ranged from $1.27 \mathrm{gm}$ (2012/AMVAR-2) to 2.84 gm (Arun) with an overall mean of $1.88 \mathrm{gm}$. higher leaf weight was recorded in Arun (2.84 gm). The stem weight ranged from $7.38 \mathrm{gm}$ (2012/AMVAR1) to $11.49 \mathrm{gm}$ (CG Amaranthus-1) with an overall mean of $8.79 \mathrm{gm}$. higher stem weight was recorded in CG Amaranthus-1 (11.49 $\mathrm{gm})$. The stem girth ranged from $1.47 \mathrm{~cm}$ (2012/AMVAR-2) to $2.39 \mathrm{~cm}$ (CG Amaranthus-1) with an overall mean of 1.66 $\mathrm{cm}$. higher stem girth was recorded in $\mathrm{CG}$ Amaranthus-1 $(2.39 \mathrm{~cm})$. The internodal length ranged from $1.36 \mathrm{~cm}$ (2012/AMVAR1) to $2.51 \mathrm{~cm}$ (Arka Saguna) with an overall mean of $1.78 \mathrm{~cm}$. higher intermodal length was recorded in Arka Saguna $(2.51 \mathrm{~cm})$. The petiole length ranged from $2.50 \mathrm{~cm}$ (2012/AMVAR-1) to $3.70 \quad \mathrm{~cm}$ (2012/AMVAR-4) with an overall mean of $3.14 \mathrm{~cm}$. Higher petiole length was recorded in 2012/AMVAR-4 $(3.70 \mathrm{~cm})$. The panicle length ranged from $9.75 \mathrm{~cm}$ (CG Amaranthus1) to $14.17 \mathrm{~cm}$ (2012/AMVAR-1) with an overall mean of $12.99 \mathrm{~cm}$. Higher panicle length was recorded in 2012/AMVAR-1 $(14.17 \mathrm{~cm})$. The number of cutting ranged from 3.75 (2012/AMVAR-7) to 5.75 (CG Amaranthus-1) with an overall mean of 4.40. Higher number of cutting was recorded in CG Amaranthus-1 (5.75). The 1000 seed weight ranged from $0.73 \mathrm{gm}$ (2012/AMVAR-5) to 0.85 gm (2012/AMVAR-6) with an overall mean of $0.79 \mathrm{gm}$. Higher 1000 seed weight was recorded in 2012/AMVAR-6 (0.85 gm). The seed yield per plant ranged from $8.52 \mathrm{gm}$ (2012/AMVAR-2) to 11.54 gm 
(2012/AMVAR-1) with an overall mean of $10.18 \mathrm{gm}$. Higher seed yield per plant was recorded in 2012/AMVAR-2 (11.54 gm). The crop duration ranged from 100.25 days (2012/AMVAR-5) to 122.50 days (2012/AMVAR-4) with an overall mean of 117.48. Higher crop duration was recorded in 2012/AMVAR-4 (122.50 days). The leaf yield ranged from $10.13 \mathrm{~kg} / \mathrm{plot}$ (Arun) to $17.83 \mathrm{~kg} / \mathrm{plot}$ (2012/AMVAR-4) with an overall mean of $14.87 \mathrm{~kg} / \mathrm{plot}$. Higher leaf yield was recorded in 2012/AMVAR-4 (17.83 $\mathrm{kg})$.

Genotypic and phenotypic coefficients of variation of different characters are presented in table 3. High magnitude of genotypic as well as phenotypic coefficient of variations were recorded for traits viz., leaf weight (35.01 and 35.63), leaf length (19.69 and 24.50) and intermodal length (18.46 and 22.99) suggested the substantial improvement on amaranthus through selection for these traits. Moderate GCV and PCV were recorded for petiole length (13.59 and 14.46), stem weight (13.44 and 14.47), leaf width (13.36 and 15.12), leaf yield per plot (13.31 \& 21.67), number of cutting (12.79 and 20.42) and number of leafs per plant (11.48 and 13.15) suggested existence of considerable variability in the population. The traits which showed high phenotypic and genotypic coefficient of variations are of economic importance and there is scope for improvement of these traits through selection. The present investigation high magnitude of heritability was recorded for most of characters. The highest heritability was recorded for the characters leaf weight (96.6\%) stem weight (86.2\%), petiole length $(86.1 \%)$, leaf width $(78.1 \%)$ and number of leaf per plant $(76.2 \%)$. The moderate heritability was observed for leaf length (64.5\%), intermodal length $(64.5 \%)$ and Low heritability was observed for 1000 seed weight $(38.5 \%)$, panicle length $(38.1 \%)$, leaf yield $\mathrm{kg}$ per plot $(37.7 \%)$, number of cutting $(37.7 \%)$, seed yield per plant $(37.0 \%)$, plant weight $(27.4 \%)$, plant height $(26.8 \%)$ and crop duration $(26.2 \%)$. The heritability value alone however, provides no indication of the amount of genetic improvement that would result from selection of superior genotypes. The heritability estimates would be reliable if it is limited in broad sense, additive and nonadditive gene effect are accompanied with high genetic advance. To facilitate the comparison of progress in various characters of different genotypes genetic advance was calculated as percentage of mean. Genetic advance as percentage of mean was observed high for leaf weight $(71.2 \%)$, leaf length (32.5\%), stem girth $(30.3 \%)$, leaf width $(25.7 \%)$, intermodal length $(25.7 \%)$ and number of leafs per plant $(20.6 \%)$. The high value of genetic advance for these traits showed that these characters are governed by additive genes and selection will be rewarding for the further improvement of such traits. Moderate genetic advance for the traits suggest that both the additive and nonadditive variance are operating in these traits.

Heritability estimates along with genetic advance are more useful than the heritability value alone for selecting the best individual. High heritability coupled with high genetic advance was observed for number of leafs per plant, leaf width and leaf weight indicating that most likely the heritability is due to additive gene effects and selection may be effective. Therefore, selection based on phenotypic performance of these traits would be effective to select desirable genotypes and Ahammed et al., (2012) were also estimated higher heritability for leaf weight per plant $(91.10 \%)$ and stem weight per plant $(82.56 \%)$.

Genotypic and phenotypic coefficients among 16 quantitative characters are presented in table 2. Significant and positive correlation of leaf yield was observed with plant height, 
plant weight, stem girth and seed yield at genotypic level. Similarly Anuja (2012) reported that plant height and stem girth are significant positive correction with leaf yield.

Plant height showed positive and significant correlation with number of leaves per plant, stem weight, stem girth, 1000 seed weight, seed yield and significant negative correction with petiole length and panicle length at genotypic level only. Plant weight showed positive and significant correlation with leaf yield at genotypic level only.

Number of leaves per plant showed positive and significant correlation with leaf length at phenotypic level only whereas, significant positively correlated with plant height, leaf weight, stem weight, stem girth and number of cutting and its significant negatively correlated with panicle length and crop duration at genotypic level only. Leaf length exhibited significant positive correlation with leaf weight at both genotypic and phenotypic level while significant positive correlated with number of leaves per plant, leaf width, internodal length, petiole length and significant negatively correlated with seed yield and leaf yield at genotypic level only.

Leaf width had positive and significant correlation with leaf length and 1000 seed weight at genotypic level only.

Leaf weight showed positive and significant correlation with leaf length and number of cutting at both genotypic and phenotypic level while significant positively correlated with number of leaves per plant at genotypic level only.

Stem weight showed positive and significant correlation with stem girth at both genotypic and phenotypic level while positive and significant correlation with plant height, number of leaves per plant, number of cutting and significant negatively correlated with panicle length at genotypic level only.

Table.1 Analysis of variance for leaf yield and its component characters in Amaranthus

\begin{tabular}{|c|c|c|c|c|}
\hline \multirow[b]{2}{*}{ S. NO. } & \multirow[b]{2}{*}{$\begin{array}{c}\text { Character } \\
\text { (df) }\end{array}$} & \multicolumn{3}{|c|}{ Mean sums of square } \\
\hline & & $\begin{array}{c}\text { Replication } \\
3\end{array}$ & $\begin{array}{c}\text { Treatment } \\
9\end{array}$ & $\begin{array}{c}\text { Error } \\
27\end{array}$ \\
\hline 1 & Plant height (cm) & 5.674 & $5.862 *$ & 2.381 \\
\hline 2 & Plant weight (gm) & 46.667 & $133.489 *$ & 53.148 \\
\hline 3 & Number of leafs/plant & 0.899 & $24.774 * *$ & 1.795 \\
\hline 4 & Leaf length $(\mathrm{cm})$ & 0.454 & $6.588 * *$ & 0.802 \\
\hline 5 & Leaf width (cm) & 0.753 & $1.172 *$ & 0.514 \\
\hline 6 & Leaf weight (gm) & 0.008 & $1.756^{* *}$ & 0.015 \\
\hline 7 & Stem weight (gm) & 0.137 & $5.808 * *$ & 0.223 \\
\hline 8 & Stem girth $(\mathbf{c m})$ & 0.036 & $0.320 * *$ & 0.025 \\
\hline 9 & Internodal length $(\mathrm{cm})$ & 0.018 & $0.492 * *$ & 0.060 \\
\hline 10 & Petiole length $(\mathrm{cm})$ & 0.061 & $0.874 * *$ & 0.107 \\
\hline 11 & Panicle length $(\mathrm{cm})$ & 0.975 & $8.920 * *$ & 2.578 \\
\hline 12 & Number of cutting & 0.467 & $1.789 * *$ & 0.522 \\
\hline 13 & 1000 seed weight (gm) & 0.005 & $0.006 * *$ & 0.002 \\
\hline 14 & Seed yield (gm/ plant) & 3.811 & $4.776 * *$ & 1.428 \\
\hline 15 & Crop duration & 6.958 & $170.081 *$ & 70.236 \\
\hline 16 & Leaf yield (kg/plot) & 92.737 & $22.131 * *$ & 6.468 \\
\hline
\end{tabular}

$*$ : Significant at 5\%, **: Significant at $1 \%$ 
Table.2 Mean performance for leaf yield and its components in Amaranthus

\begin{tabular}{|c|c|c|c|c|c|c|c|c|c|c|c|c|c|c|c|c|}
\hline $\begin{array}{l}\text { Characters } \\
\text { Genotypes }\end{array}$ & $\begin{array}{c}\text { Plant } \\
\text { height } \\
(\mathbf{c m})\end{array}$ & $\begin{array}{c}\text { Plant } \\
\text { weight } \\
\text { (gm) }\end{array}$ & $\begin{array}{c}\text { Number } \\
\text { of leafs/ } \\
\text { plant }\end{array}$ & $\begin{array}{c}\text { Leaf } \\
\text { length } \\
(\mathrm{cm})\end{array}$ & $\begin{array}{c}\text { Leaf } \\
\text { width } \\
(\mathrm{cm})\end{array}$ & $\begin{array}{c}\text { Leaf } \\
\text { weight } \\
\text { (gm) }\end{array}$ & $\begin{array}{c}\text { Stem } \\
\text { weight } \\
\text { (gm) }\end{array}$ & $\begin{array}{l}\text { Stem } \\
\text { girth } \\
(\mathbf{c m})\end{array}$ & $\begin{array}{r}\text { Internodal } \\
\text { length }(\mathbf{c m})\end{array}$ & $\begin{array}{c}\text { Petiole } \\
\text { length } \\
\text { (cm) }\end{array}$ & $\begin{array}{c}\text { Panicle } \\
\text { length } \\
(\mathrm{cm})\end{array}$ & $\begin{array}{l}\text { Number } \\
\text { of cutting }\end{array}$ & $\begin{array}{c}1000 \\
\text { seed } \\
\text { weight } \\
(\mathrm{gm})\end{array}$ & $\begin{array}{l}\text { Seed } \\
\text { yield } \\
\text { (gm/ } \\
\text { plant) }\end{array}$ & $\begin{array}{c}\text { Crop } \\
\text { duration } \\
\text { (Days) }\end{array}$ & $\begin{array}{c}\text { Leaf } \\
\text { yield } \\
(\mathrm{kg} / \mathrm{plot})\end{array}$ \\
\hline 2012/AMVAR-1 & 10.49 & 52.50 & 20.52 & 4.83 & 3.45 & 1.66 & 7.38 & 1.54 & 1.36 & 2.50 & 14.17 & 4.25 & 0.77 & 11.54 & 120.50 & 14.67 \\
\hline 2012/AMVAR-2 & 8.39 & 62.50 & 18.87 & 5.75 & 4.38 & 1.27 & 8.50 & 1.47 & 1.39 & 3.39 & 13.95 & 4.25 & 0.80 & 8.52 & 118.50 & 13.61 \\
\hline 2012/AMVAR-3 & 9.61 & 65.25 & 18.61 & 5.33 & 4.05 & 1.39 & 8.27 & 1.54 & 1.58 & 2.68 & 12.94 & 4.25 & 0.82 & 10.83 & 121.50 & 14.69 \\
\hline 2012/AMVAR-4 & 9.77 & 62.50 & 19.98 & 6.15 & 4.50 & 1.48 & 8.14 & 1.69 & 2.21 & 3.70 & 14.08 & 4.00 & 0.74 & 11.02 & 122.50 & 17.83 \\
\hline 2012/AMVAR-5 & 9.06 & 70.00 & 23.79 & 5.57 & 3.82 & 1.67 & 8.33 & 1.49 & 1.72 & 3.43 & 12.71 & 4.00 & 0.73 & 9.44 & 100.25 & 15.84 \\
\hline 2012/AMVAR-6 & 10.39 & 56.75 & 18.56 & 5.37 & 4.24 & 1.42 & 8.13 & 1.49 & 1.84 & 3.47 & 11.63 & 4.00 & 0.85 & 10.98 & 119.25 & 13.56 \\
\hline 2012/AMVAR-7 & 11.97 & 70.50 & 20.67 & 5.35 & 4.04 & 1.48 & 10.13 & 1.57 & 1.67 & 2.71 & 13.77 & 3.75 & 0.83 & 9.91 & 120.50 & 17.41 \\
\hline Arka saguna & 9.17 & 64.75 & 19.83 & 7.78 & 5.00 & 2.82 & 9.34 & 1.55 & 2.51 & 3.38 & 12.19 & 5.50 & 0.78 & 9.35 & 121.00 & 13.62 \\
\hline Arrun & 9.99 & 57.00 & 21.63 & 8.98 & 4.98 & 2.84 & 8.23 & 1.86 & 1.84 & 3.59 & 14.70 & 4.25 & 0.77 & 8.89 & 116.00 & 10.13 \\
\hline CG Amaranthus-1 & 12.15 & 60.25 & 26.36 & 6.30 & 5.06 & 2.82 & 11.49 & 2.39 & 1.72 & 2.57 & 9.75 & 5.75 & 0.81 & 11.37 & 114.75 & 17.36 \\
\hline Mean (x) & 10.10 & 62.20 & 20.88 & 6.14 & 4.35 & 1.88 & 8.79 & 1.66 & 1.78 & 3.14 & 12.99 & 4.40 & 0.79 & 10.18 & 117.48 & 14.87 \\
\hline SEm \pm & 0.771 & 3.645 & 0.670 & 0.448 & 0.359 & 0.062 & 0.236 & 0.079 & 0.122 & 0.164 & 0.803 & 0.361 & 0.021 & 0.597 & 4.190 & 9.927 \\
\hline $\mathrm{CD}(\mathrm{p}=\mathbf{0 . 0 5})$ & 2.2386 & 10.5772 & 1.9440 & 1.2994 & 1.0406 & 0.1797 & 0.6856 & 0.228 & 0.3542 & 0.4747 & 2.3296 & 1.0485 & 0.0599 & 1.7337 & 12.1592 & 3.69 \\
\hline CV (\%) & 15.28 & 11.721 & 6.417 & 14.589 & 16.485 & 6.576 & 5.373 & 9.476 & 13.702 & 10.421 & 12.363 & 16.424 & 5.235 & 11.735 & 7.134 & 17.104 \\
\hline
\end{tabular}

Table.3 Genetic parameter of variability for leaf yield and its component character in Amaranthu

\begin{tabular}{|c|c|c|c|c|c|c|c|c|}
\hline \multirow[t]{2}{*}{ S.No. } & \multirow[t]{2}{*}{ Characters } & \multirow[t]{2}{*}{ Mean } & \multicolumn{2}{|c|}{ Range } & \multicolumn{2}{|c|}{$\begin{array}{c}\text { Coefficient of } \\
\text { Variation (\%) }\end{array}$} & \multirow{2}{*}{$\begin{array}{l}\text { Heritability } \\
\quad\left(h^{2} \%\right)\end{array}$} & \multirow{2}{*}{$\begin{array}{c}\text { GA as percent } \\
\text { of Mean }\end{array}$} \\
\hline & & & Min $^{m}$ & $\operatorname{Max}^{\mathbf{m}}$ & GCV & PCV & & \\
\hline 1 & Plant height (cm) & 10.10 & 8.39 & 12.15 & 9.24 & 17.87 & 26.8 & 9.8 \\
\hline 2 & Plant weight (gm) & 62.20 & 52.50 & 70.50 & 7.21 & 13.76 & 27.4 & 7.76 \\
\hline 3 & Number of leafs/plant & 20.88 & 18.56 & 26.36 & 11.48 & 13.15 & 76.2 & 20.6 \\
\hline 4 & Leaf length $(\mathrm{cm})$ & 6.14 & 4.83 & 8.98 & 19.69 & 24.50 & 64.5 & 32.5 \\
\hline 5 & Leaf width (cm) & 4.35 & 3.45 & 5.06 & 13.36 & 15.12 & 78.1 & 25.7 \\
\hline 6 & Leaf weight (gm) & 1.88 & 1.27 & 2.84 & 35.01 & 35.63 & 96.6 & 71.2 \\
\hline 7 & Stem weight (gm) & 8.79 & 7.38 & 11.49 & 13.44 & 14.47 & 86.2 & 11.4 \\
\hline 8 & Stem girth $(\mathbf{c m})$ & 1.66 & 1.47 & 2.39 & 9.55 & 15.46 & 38.1 & 30.3 \\
\hline 9 & Internodal length $(\mathrm{cm})$ & 1.78 & 1.36 & 2.51 & 18.46 & 22.99 & 64.5 & 25.7 \\
\hline 10 & Petiole length (cm) & 3.14 & 2.50 & 3.70 & 13.59 & 14.65 & 86.1 & 12.3 \\
\hline 11 & Panicle length (cm) & 12.99 & 9.75 & 14.70 & 9.60 & 15.71 & 38.1 & 16.1 \\
\hline 12 & Number of cutting & 4.40 & 3.75 & 5.75 & 12.79 & 20.42 & 37.7 & 16.8 \\
\hline 13 & 1000 seed weight (gm) & 0.79 & 0.73 & 0.85 & 4.14 & 6.68 & 38.5 & 5.06 \\
\hline 14 & Seed yield (gm/ plant) & 10.18 & 8.52 & 11.54 & 8.99 & 14.78 & 37.0 & 11.2 \\
\hline 15 & Crop duration & 117.48 & 100.25 & 122.50 & 4.25 & 8.31 & 26.2 & 4.4 \\
\hline 16 & Leaf yield (kg/plot) & 14.87 & 10.13 & 17.83 & 13.31 & 21.67 & 37.7 & 16.8 \\
\hline
\end{tabular}


Table.4 Genotypic and phenotypic correlation coefficient between leaf yield and its component

\begin{tabular}{|c|c|c|c|c|c|c|c|c|c|c|c|c|c|c|c|c|c|}
\hline \multicolumn{2}{|c|}{ Character } & $\begin{array}{c}01 . \\
\text { Plant } \\
\text { height } \\
(\mathrm{cm})\end{array}$ & $\begin{array}{c}02 . \\
\text { Plant } \\
\text { weight } \\
(\mathrm{gm})\end{array}$ & $\begin{array}{c}03 . \\
\text { Number } \\
\text { of leafs/ } \\
\text { plant }\end{array}$ & $\begin{array}{c}04 . \\
\text { Leaf } \\
\text { length } \\
(\mathrm{cm})\end{array}$ & $\begin{array}{c}05 . \\
\text { Leaf } \\
\text { width } \\
(\mathrm{cm})\end{array}$ & $\begin{array}{c}\text { 06. } \\
\text { Leaf } \\
\text { weight } \\
(\mathrm{gm})\end{array}$ & $\begin{array}{c}07 . \\
\text { Stem } \\
\text { weight } \\
(\mathrm{gm})\end{array}$ & $\begin{array}{l}\mathbf{0 8 .} \\
\text { Stem } \\
\text { girth } \\
(\mathrm{cm})\end{array}$ & $\begin{array}{c}09 . \\
\text { Internodal } \\
\text { length } \\
(\mathbf{c m})\end{array}$ & $\begin{array}{c}10 . \\
\text { Petiole } \\
\text { length } \\
(\mathrm{cm})\end{array}$ & $\begin{array}{c}11 . \\
\text { Panicle } \\
\text { length } \\
(\mathrm{cm})\end{array}$ & $\begin{array}{c}12 . \\
\text { Number } \\
\text { of } \\
\text { cutting }\end{array}$ & $\begin{array}{c}13 . \\
1000 \\
\text { seed } \\
\text { weight } \\
(\mathrm{gm}) \\
\end{array}$ & $\begin{array}{c}14 . \\
\text { Seed } \\
\text { yield } \\
\text { (gm/ } \\
\text { plant) }\end{array}$ & $\begin{array}{c}15 . \\
\text { Crop } \\
\text { duration }\end{array}$ & $\begin{array}{c}16 . \\
\text { Leaf } \\
\text { yield } \\
(\mathrm{kg} / \mathrm{plot})\end{array}$ \\
\hline \multirow{2}{*}{1} & $\mathbf{P}$ & 1.000 & 0.025 & 0.193 & -0.127 & 0.146 & 0.106 & 0.529 & 0.503 & 0.082 & -0.354 & -0.149 & 0.122 & 0.150 & 0.315 & 0.024 & 0.326 \\
\hline & $\mathbf{G}$ & 1.000 & -0.238 & $0.703^{*}$ & -0.175 & 0.424 & 0.320 & $0.747 * *$ & $0.783 * *$ & -0.297 & $-0.872 * *$ & $-0.631 *$ & 0.225 & $0.720 * *$ & $0.814 * *$ & 0.324 & $0.569 *$ \\
\hline \multirow{2}{*}{2} & $\mathbf{P}$ & & 1.000 & -0.056 & -0.039 & -0.204 & -0.155 & 0.246 & -0.023 & 0.154 & 0.049 & -0.019 & -0.036 & -0.096 & -0.227 & --0.264 & 0.166 \\
\hline & G & & 1.000 & 0.206 & -0.183 & -0.322 & -0.229 & 0.447 & -0.326 & 0.221 & 0.067 & -0.082 & -0.230 & -0.073 & -0.543 & 0.414 & $0.735 * *$ \\
\hline \multirow{2}{*}{3} & $\mathbf{P}$ & & & 1.000 & $0.990 * *$ & 0.060 & 0.488 & 0.479 & 0.424 & -0.069 & -0.204 & -0.304 & 0.222 & -0.208 & 0.032 & -0.313 & 0.236 \\
\hline & G & & & 1.000 & 0.172 & 0.080 & $0.582^{*}$ & $0.669^{*}$ & $0.918 * *$ & -0.029 & -0.241 & $-0.632 *$ & $0.641 *$ & -0.337 & 0.187 & $-0.898 * *$ & 0.387 \\
\hline \multirow{2}{*}{4} & $\mathbf{P}$ & & & & 1.000 & 0.406 & $0.657 *$ & 0.106 & 0.077 & 0.476 & 0.408 & 0.078 & 0.257 & -0.191 & -0.378 & -0.013 & -0.344 \\
\hline & G & & & & 1.000 & $0.645^{*}$ & $0.869 * *$ & 0.142 & -0.041 & $0.614 *$ & $0.581 *$ & 0.118 & 0.491 & -0.374 & -0.607 & 0.007 & $-0.746 * *$ \\
\hline \multirow{2}{*}{5} & $\mathbf{P}$ & & & & & 1.000 & 0.474 & 0.258 & 0.168 & 0.195 & 0.263 & -0.154 & 0.181 & 0.353 & -0.172 & 0.145 & -0.376 \\
\hline & G & & & & & 1.000 & 0.503 & 0.359 & 0.362 & 0.300 & 0.306 & -0.477 & 0.334 & $0.556^{*}$ & -0.019 & 0.185 & -0.487 \\
\hline \multirow[b]{2}{*}{6} & $\mathbf{P}$ & & & & & & 1.000 & 0.428 & 0.296 & 0.374 & 0.090 & -0.264 & $0.580^{*}$ & -0.116 & -0.101 & -0.066 & -0.236 \\
\hline & $\mathbf{G}$ & & & & & & 1.000 & 0.487 & 0.528 & 0.510 & 0.093 & -0.398 & $0.900 * *$ & -0.228 & -0.176 & -0.151 & -0.356 \\
\hline \multirow{2}{*}{7} & $\mathbf{P}$ & & & & & & & 1.000 & $0.636^{*}$ & 0.156 & -0.324 & -0.442 & 0.381 & 0.147 & 0.001 & -0.031 & 0.339 \\
\hline & G & & & & & & & 1.000 & $0.869 * *$ & 0.149 & -0.353 & $-0.803 * *$ & $0.778 * *$ & 0.457 & 0.080 & -0.072 & 0.519 \\
\hline \multirow{2}{*}{8} & $\mathbf{P}$ & & & & & & & & 1.000 & 0.119 & -0.362 & -0.380 & 0.322 & 0.149 & 0.200 & -0.067 & 0.267 \\
\hline & G & & & & & & & & 1.000 & -0.151 & -0.504 & $-0.974 * *$ & $0.953 * *$ & 0.226 & $0.641 *$ & 0.002 & $0.602 *$ \\
\hline \multirow{2}{*}{9} & $\mathbf{P}$ & & & & & & & & & 1.000 & 0.362 & -0.099 & 0.329 & -0.169 & -0.063 & 0.122 & 0.055 \\
\hline & G & & & & & & & & & 1.000 & 0.508 & -0.233 & 0.390 & -0.356 & -0.115 & 0.182 & -0.014 \\
\hline \multirow{2}{*}{10} & $\mathbf{P}$ & & & & & & & & & & 1.000 & 0.222 & -0.044 & -0.168 & -0.442 & -0.180 & -0.388 \\
\hline & G & & & & & & & & & & 1.000 & 0.213 & -0.268 & -0.418 & $-0.791 * *$ & -0.347 & $-0.607 *$ \\
\hline \multirow{2}{*}{11} & $\mathbf{P}$ & & & & & & & & & & & 1.000 & -0.346 & -0.152 & -0.329 & -0.038 & -0.234 \\
\hline & $\mathbf{G}$ & & & & & & & & & & & 1.000 & $-0.972 * *$ & $-0.576^{*}$ & -0.424 & 0.468 & -0.380 \\
\hline \multirow{2}{*}{12} & $\mathbf{P}$ & & & & & & & & & & & & 1.000 & 0.084 & 0.226 & -0.013 & 0.008 \\
\hline & $\mathbf{G}$ & & & & & & & & & & & & 1.000 & 0.017 & -0.014 & 0.088 & 0.024 \\
\hline \multirow{2}{*}{13} & $\mathbf{P}$ & & & & & & & & & & & & & 1.000 & 0.206 & 0.243 & -0.085 \\
\hline & G & & & & & & & & & & & & & 1.000 & 0.195 & $0.647 *$ & 0.012 \\
\hline \multirow{2}{*}{14} & $\mathbf{P}$ & & & & & & & & & & & & & & 1.000 & 0.066 & 0.348 \\
\hline & G & & & & & & & & & & & & & & 1.000 & 0.526 & $0.655^{*}$ \\
\hline \multirow{2}{*}{15} & $\mathbf{P}$ & & & & & & & & & & & & & & & 1.000 & -0.083 \\
\hline & G & & & & & & & & & & & & & & & 1.000 & 0.039 \\
\hline \multirow{2}{*}{16} & $\mathbf{P}$ & & & & & & & & & & & & & & & & 1.000 \\
\hline & G & & & & & & & & & & & & & & & & 1.000 \\
\hline
\end{tabular}


Table.5 Direct and indirect effect of component character on leaf yield in Amaranthus

\begin{tabular}{|c|c|c|c|c|c|c|c|c|c|c|c|c|c|c|c|c|}
\hline Character & $\begin{array}{l}\text { 01. } \\
\text { Plant } \\
\text { height } \\
(\mathrm{cm})\end{array}$ & $\begin{array}{c}02 . \\
\text { Plant } \\
\text { weight } \\
\text { (gm) }\end{array}$ & $\begin{array}{c}03 . \\
\text { Number } \\
\text { of leafs/ } \\
\text { Plant }\end{array}$ & $\begin{array}{c}04 . \\
\text { Leaf } \\
\text { length } \\
(\mathrm{cm})\end{array}$ & $\begin{array}{c}05 . \\
\text { Leaf } \\
\text { width } \\
(\mathrm{cm})\end{array}$ & $\begin{array}{c}\text { 06. } \\
\text { Leaf } \\
\text { weight } \\
(\mathrm{gm})\end{array}$ & $\begin{array}{c}07 . \\
\text { Stem } \\
\text { weight } \\
(\mathrm{gm})\end{array}$ & $\begin{array}{l}\mathbf{0 8 .} \\
\text { Stem } \\
\text { girth } \\
(\mathrm{cm})\end{array}$ & $\begin{array}{l}09 . \\
\text { Internodal } \\
\text { length } \\
(\mathbf{c m})\end{array}$ & $\begin{array}{c}10 . \\
\text { Petiole } \\
\text { length } \\
(\mathrm{cm})\end{array}$ & $\begin{array}{c}11 . \\
\text { Panicle } \\
\text { length } \\
(\mathrm{cm})\end{array}$ & $\begin{array}{c}12 . \\
\text { Number } \\
\text { of } \\
\text { cutting }\end{array}$ & $\begin{array}{c}13 . \\
1000 \\
\text { seed } \\
\text { weight } \\
\text { (gm) }\end{array}$ & $\begin{array}{c}14 . \\
\text { Seed } \\
\text { yield } \\
\text { (gm/ } \\
\text { plant) }\end{array}$ & $\begin{array}{c}15 . \\
\text { Crop } \\
\text { duration }\end{array}$ & $\begin{array}{l}16 . \\
\text { Leaf } \\
\text { yield } \\
\text { (kg/plot) }\end{array}$ \\
\hline Plant height $(\mathrm{cm})$ & -0.110 & 0.015 & 0.317 & -0.027 & -0.115 & -0.416 & 0.961 & 0.096 & -0.131 & -0.038 & -0.179 & -0.016 & -0.236 & 0.395 & 0.053 & 0.569 \\
\hline Plant weight (gm) & 0.026 & -0.062 & 0.093 & -0.028 & 0.087 & 0.298 & 0.575 & -0.040 & 0.097 & 0.003 & -0.023 & 0.016 & 0.024 & -0.264 & -0.068 & 0.735 \\
\hline Number of leafs/plant & -0.077 & -0.013 & 0.450 & 0.026 & -0.022 & -0.758 & 0.861 & 0.113 & -0.013 & -0.011 & -0.179 & -0.046 & 0.110 & 0.091 & -0.147 & 0.387 \\
\hline Leaf length (cm) & 0.019 & 0.011 & 0.077 & 0.152 & -0.175 & -1.131 & 0.183 & -0.005 & 0.270 & 0.026 & 0.033 & -0.035 & 0.122 & -0.295 & 0.001 & -0.746 \\
\hline Leaf width (cm) & -0.047 & 0.020 & 0.036 & 0.098 & -0.271 & -0.655 & 0.462 & 0.044 & 0.132 & 0.013 & -0.135 & -0.024 & -0.182 & -0.009 & 0.030 & -0.487 \\
\hline Leaf weight (gm) & -0.035 & 0.014 & 0.262 & 0.132 & -0.136 & -1.301 & 0.627 & 0.065 & 0.224 & 0.004 & -0.113 & -0.064 & 0.075 & -0.085 & -0.025 & -0.356 \\
\hline Stem weight (gm) & -0.082 & -0.028 & 0.301 & 0.022 & -0.097 & -0.634 & 1.287 & 0.107 & 0.065 & -0.016 & -0.227 & -0.055 & -0.150 & 0.039 & -0.012 & 0.519 \\
\hline Stem girth $(\mathbf{c m})$ & -0.086 & 0.020 & 0.413 & -0.006 & -0.098 & -0.687 & 1.119 & 0.123 & -0.067 & -0.022 & -0.276 & -0.068 & -0.074 & 0.311 & 0.000 & 0.602 \\
\hline Internodel length $(\mathrm{cm})$ & 0.033 & -0.014 & -0.013 & 0.093 & -0.081 & -0.663 & 0.191 & -0.019 & 0.440 & 0.022 & -0.066 & -0.028 & 0.117 & -0.056 & 0.030 & -0.014 \\
\hline Petiole length (cm) & 0.096 & -0.004 & -0.109 & 0.088 & -0.083 & -0.122 & -0.454 & -0.062 & 0.223 & 0.044 & 0.060 & 0.019 & 0.137 & -0.384 & -0.057 & -0.607 \\
\hline Panicle length (cm) & 0.069 & 0.005 & -0.285 & 0.018 & 0.129 & 0.518 & -1.034 & -0.120 & -0.102 & 0.009 & 0.283 & 0.069 & 0.189 & -0.206 & 0.077 & -0.380 \\
\hline Number of cutting & -0.025 & 0.014 & 0.289 & 0.074 & -0.090 & -1.171 & 1.001 & 0.117 & 0.171 & -0.012 & -0.275 & -0.071 & -0.006 & -0.007 & 0.014 & 0.024 \\
\hline 1000 seed weight $(\mathrm{gm})$ & -0.079 & 0.005 & -0.152 & -0.057 & -0.151 & 0.297 & 0.588 & 0.028 & -0.157 & -0.018 & -0.163 & -0.001 & -0.328 & 0.095 & 0.106 & 0.012 \\
\hline Seed yield (gm/plant) & -0.089 & 0.034 & 0.084 & -0.092 & 0.005 & 0.229 & 0.103 & 0.079 & -0.051 & -0.035 & -0.120 & 0.001 & -0.064 & 0.486 & 0.086 & 0.655 \\
\hline Crop duration & -0.036 & 0.026 & -0.404 & 0.001 & -0.050 & 0.197 & -0.093 & 0.000 & 0.080 & -0.015 & 0.133 & -0.006 & -0.212 & 0.255 & 0.163 & 0.039 \\
\hline
\end{tabular}


Stem girth showed positive and significant correlation with stem weight at both genotypic and phenotypic level while positive and significant correlation with plant height, number of leaves, number of cutting, seed yield and leaf yield and significant negatively correlated with panicle length at genotypic level only. Internodal length exhibited positive and significant correction with leaf length at genotypic level only (Table 4).

Petiole length showed significant positive correlation with leaf length while negative and significant correlation with seed yield per plot and leaf yield at genotypic level. Panicle length showed significant negative correlation with plant height, number of leaves per plant, number of cutting and 1000 seed weight at genotypic level only (Plant 5).

Number of cutting had positive and significant correlation with leaf weight at both genotypic and phenotypic level while positive and significant correlation with number of leaves per plant, stem weight and stem girth and it is significant negatively correlated with panicle length at genotypic level only.

1000 seed weight exhibited positive and significant correlation with plant height, leaf weight and crop duration while significant negatively correlated with panicle length at genotypic level only.

Seed yield per plant had positive and significant correlation with plant height, stem girth and leaf yield while significant negative correlated with leaf length and petiole length at genotypic level only. Crop duration had positive and significant correlation with 1000 seed weight while significant negatively correlated with number of leaves per plant at genotypic level only.

Path coefficient analysis is an important tool for partitioning the correlation coefficients into the direct and indirect effects of independent variables on a dependent variable. In this analysis, leaf yield was taken as dependent variable and the rest of the characters were considered as independable variables.

The path coefficient analysis which splits total correlation coefficient of different characters into direct and indirect effects on leaf yield $\mathrm{kg}$ per plot in such a manner that the sum of direct and indirect effects is equal to total genotypic correlation as presented in table 3 .

The data revealed that stem weight showed the highest positive direct effect (1.287) on leaf yield and indirect positive effected through number of leaves, leaf length, stem girth, internodal length, seed yield, whereas it was negative effect through plant height, plant weight, leaf width, leaf weight, petiole length, panicle length, number of cutting, 1000 seed weight, crop duration. Similarly Aruna (2010) reported that stem weight direct effect on leaf yield.

Plant height showed direct negative effect on leaf yield and indirect positive effect through plant weight, number of leaves, stem girth, stem weight seed yield, crop duration, whereas it was negative effect through leaf length, petiole length, panicle length, leaf width, leaf weight, internodal length, number of cutting, 1000 seed weight. Kendre et al., (2013) also finding that plant height had the direct negative effect on leaf yield.

Plant weight had direct negative effect on leaf yield and this trait indirect positive effect through plant height, number of leaves, leaf width, leaf weight, stem weight, internodal length, panicle length, number of cutting, 1000 seed weight, whereas its effect was negative leaf length, stem girth, panicle length, seed yield, crop duration. 
Number of leaves had exhibited positive direct effect on leaf yield and this trait was influenced indirect positive effect through leaf length, stem weight, stem girth, 1000 seed weight, seed yield, whereas negative effect through leaf width, leaf weight, inetrnodal length, number of cutting, crop duration.

Leaf length had direct positive effect on leaf yield and it was also exerted indirect positive effects through plant height, plant weight, number of leaves, stem weight, internodal length, panicle length, petiole length, 1000 seed weight, crop duration, whereas negative effect through leaf width, leaf weight stem girth, number of cutting, seed yield.

Leaf width had negative direct effect towards leaf yield and its indirect positive effect through plant weight, number of leaves, leaf length, stem weight, stem girth, internodal length, panicle length, crop duration, whereas it was indirect negative effect through plant height, leaf weight, panicle length, number of cutting, 1000 seed weight, seed yield.

Leaf weight showed highest direct negative effect on leaf yield and its indirect positive effect through plant weight, number of leaves, leaf length, stem weight, stem girth, internodal length, panicle length, 1000 seed weight, whereas negative effect through plant height, leaf weight, panicle length, number of cutting, seed yield, crop duration.

Stem girth had positive direct effect on leaf yield and positive indirect effect through plant weight, number of leaves, stem weight, seed yield, whereas its negative effect through plant height, leaf length, leaf width, leaf weight, internodal length, panicle length, petiole length, number of cutting, 1000 seed weight.

Internodal length showed positive direct effect on leaf yield and positive indirect effect through plant height, leaf length, stem weight, petiole length, 1000 seed weight, crop duration, whereas its negative effect through plant weight, number of leaves, leaf weight, leaf width, stem girth, panicle length, number of cutting, seed yield.

Petiole length had exhibited positive direct effect on leaf yield and indirect positive effect through plant height, leaf length, internodal length, panicle length, number of cutting, 1000 seed weight, whereas negative effect through plant weight, number of leaves, leaf width, leaf weight, stem weight, stem girth, seed yield, crop duration.

Panicle length had positive and direct effect on leaf yield and indirect positive effect through plant height, plant weight, leaf length, leaf width, leaf weight, petiole length, number of cutting, 1000 seed weight, crop duration, whereas it was negative effect through number of leaves, stem weight, stem girth, internodal length, seed yield.

Number of cutting had direct negative effect on leaf yield and its indirect positive effect through plant weight, number of leaves, leaf length, stem weight, stem girth, internodal length, crop duration, whereas indirect negative effect through plant height, leaf width, leaf weight, petiole length, panicle length, 1000 seed weight, seed yield.

1000 seed weight showed negative direct effect on leaf yield, whereas it was positive indirect effect through plant weight, leaf weight, stem weight, stem girth, seed yield, crop duration and negative effect through plant height, number of leaves, leaf length, leaf width, internodal length, petiole length, panicle length, number of cutting.

Seed yield had positive direct effect on leaf yield and its positive indirect effect through plant height, number of leaves, leaf length, 
leaf width, stem weight, stem girth, number of cutting, crop duration, whereas negative indirect effect through plant height, leaf length, internodal length, petiole length, panicle length, 1000 seed weight.

Crop duration had direct and positive effect on leaf yield and indirect positive effect through plant weight, leaf length, internodal length, panicle length, seed yield, whereas it was indirect negative effect through plant height, number of leaves, leaf width, stem weight, petiole length, number of cutting, 1000 seed weight.

Conclusion of this study is the analysis of variance shows that considerable variability existed among the genotypes for most of the traits showing possibilities of further genetic improvement in amaranthus. The mean performance for leaf yield $\mathrm{kg}$ per plot of 2012/AMVAR-4 was superior among all the genotype. Higher heritability estimates coupled with high genetic advance as percent of mean were observed for leaf weight, leaf width and number of leaves per plant. Correlation studies revealed that leaf yield $\mathrm{kg}$ per plot showed the highest positive and significant correlation with plant weight and seed yield per plant at genotypic level only. Path analysis revealed that stem weight was highly positive direct effected on leaf yield thus its character show the play important role for improvement programme of amaranthus vegetable.

\section{References}

Ahammed, A. U., Rahman, M. M. and Mian, M. A. K. 2012. Genetic Variability, Heritability and Correlation in Stem Amaranth (Amaranthus tricolor). Bangladesh Journal of Plant Breeding and Genetics, 25(2): 25- 32.

Anuja, S. and Mohideen, M. K. 2012. Variability, Heritability and Genetic
Advance Studies in Amaranthus (Amaranthus Spp.). The Asian Journal of Horticulture, 2 (1): 63.

Aruna, P. 2010. Correlation and Path Analysis in Amaranthus. The Asian Journal of Horticulture, 4 (2): 361-363.

Burton, G. W. 1952. Quantitative inheritance in grases. Proc. 6th Int. Grassland Cong., 1: 227-283.

Burton, G. W. and De Vane, E. H. 1953. Estimating heritability in tall fesue (Fesluca arundinacea) from replicated clonal material. Agron. J., 45: 418-481.

Dewey, D. R. and Lu, K. H. 1959. A correlation and path coefficient analysis of components of crested wheat grass seed production. Agron. J., 51: 512-515.

Johanson, H.W., Robinson, H. F. and Comstock, R. E. 1955. Estimates of genetic and environmental variability of soybean. Agron. J., 47: 314-318.

Katiyar R.S., Shukla S., Rai S., 2000. Varietal performance of grain amaranth (A. hypochondriacus) on sodic soil. Proceedings of the National Academy of Science, 70(2): 185-187.

Kendre, V., Dod, V. N., Nagre, P. K., Kale, V. S. Potdukhe, N. R. 2013. Correlation and Path Analysis in Leafy Amaranthus Tricolor L., Annals of Plant Physiology, 27 (1/2): 27- 29.

Lakshmi, B. and Vimala, V. (2000). Nutritive value of dehydrated green leafy vegetable powders. Journal of food science and technology, 37 (5): 465471.

Miller, P. A., Williams, J. E., Robinson, H. F. and Comstock, R. E. 1958. Estimates of variance and co-variance in upland cotton and their implications in selection. Agron. J., 50: 126-131.

Prakash Dhan and Pal M. 1991. Nutritional and Antinutritional Composition of vegetable and grain Amaranth leaves. J. Sci. Food Agric, 57:573-583.

Prakash Dhan, Joshi, BD and Pal M, 1995. 
Vitamin C in leaves and seed oil composition of the Amaranthus species. International J. Food Sciences and Nutrition, 46: 47-51.

Panse, V. G. and Sukhatme, P. V. (1967). Statistical methods for agricultural workers. ICAR, New Delhi: 97-151.

Raut, V. R., Dodake, S. S. and Chimote, V. P. 2014. Evaluation of genetic diversity in grain amaranth (Amaranthus hypochondriacus) at molecular level using ISSR markers. Indian Journal of Agricultural Biochemistry, 27(1):60- 65.

Shukla, Sudhir and Singh, S. P. 2000. Studies on Genetic Parameters in Vegetable Amaranth. Journal of Genetics \& Breeding, 54 (2): 133-135.

Shukla, S. and Singh, S.P. 2003. Correlation and Path Analysis in Grain Amaranth (Amaranthus Spp.). Indian J. Genet., 63 (2): 163-164.

Shukla, S., Bhargava, A., Chatterjee, A., Srivastava, A., and Singh, S. P. 2006. Genotypic variability in vegetable amaranth (A. tricolor) for foliage yield and its contributing traits over successive cuttings and years. Euphytica, 151: 103-110.

Sankaran, A. 1943. Cultivation of leafy vegetables in the northern circars, Madras. Agri. J., 31: 285-286.

Varalakshmi, B. 2004. Characterization and Preliminary Evaluation of Vegetable Amaranth (Amaranthus Spp.) Germplasm. Plant Genetic Resources Newsletter, 137: 55-57.

\section{How to cite this article:}

Ishwar Singh Diwan, Neeraj Shukla and Vivek Kurrey. 2017. Genetic Studies in Amaranthus Germplasm. Int.J.Curr.Microbiol.App.Sci. 6(8): 2459-2470. doi: https://doi.org/10.20546/ijcmas.2017.608.291 